\title{
Research on the Structural Design of Pipe Box Mechanical Components based on the Finite Element Analysis and Multimedia Visual Analysis Technology
}

\author{
Qi-zhi YU \\ $\{$ XXXX@Springer.com $\}$ \\ Vocational Technical College, Shanghai University of Engineering Science, Shanghai, China
}

\begin{abstract}
Structural design and optimization of the mechanical components are of vital importance to the economic benefit and sustainable development of the enterprises. In order to optimize the structure design of the mechanical components, the structural design of pipe box mechanical components based on the finite element analysis and multimedia visual analysis technology is proposed in this paper, the optimization process and its mathematical model based on the finite element analysis are given, and the structure and shape design of box components are optimized. The experimental results show that this scheme not only can improve the overall size and shape of the components, but also reduce the application of materials, and realize the computer aided design of the mechanical components.
\end{abstract}

Keywords: Mechanical components; structural design; finite element analysis; multimedia visual analysis.

\section{Introduction}

With the rapid development of industry, structural design and optimization of mechanical components have a vital role in the economic efficiency and sustainable development of the enterprises. Optimization design of the key parts of XK640 CNC milling machine based on the ANSYS static stiffness analysis was proposed by Yang Qiongjing in 2007, which was of great significance to improve the quality of the design of CNC machine tool components [1]. Design of detachable connection structure based on TRIZ was put forward by Liu Zhifeng in 2010, which improved the dis-assembly performance of the product components, and had high universality and feasibility [2]. The design of the reliability sensitivity of the components based on the measured information was proposed by Wang Xingang in 2012, the results shown that the research provided a theoretical basis for the design and life prediction of the mechanical components [3]. Three dimensional design of cylindrical gear based on Pro/E was researched by Huang Qiong in 2014, the results shown that the method reduced the workload, and the parametric 3D design became more intuitive and simple [4]. Research on the optimization of packaging machinery parts based on Pro/E behavior modeling technology was proposed by Zhang Hongjun in 2015, experimental results shown that the scheme improved the efficiency and quality of the design of packaging machinery in a certain extent [5]. 
However, most of the current mechanical components structure design methods can't handle a large range of structural types, the methods dealing with the problems are relatively simple, and usually consume a large amount of materials, which causes the increasing of the cost.

In order to optimize the structure design of the mechanical components, this paper presents the structure design of the pipe box mechanical components based on the finite element analysis technology. The remainder of this paper is organized as follows. Section 2 describes the relevant theories and techniques. Section 3 gives the study on the structural design of pipe box mechanical components based on the finite element analysis and multimedia visual analysis technology. Section 4 presents a real experiment to evaluate the method. Conclusion is summarized in Section 5.

\section{State of the Art}

\subsection{Finite Element Analysis}

Finite element analysis refers to use mathematical methods to simulate physical systems, and use the finite number to close to the real physical system as maximum as possible in the form of a unit [6]. The concept of finite element analysis method is clear and simple, so it is easy to be applied in all aspects of the system. On the one hand, finite element analysis method divides a whole into a plurality of units, and the nodes between each unit are regarded as a discrete point, then according to the characteristics of each unit to carry out corresponding research, which doesn't need to consider the complex differential equations [7]. On the other hand, the finite element analysis method gathers different shapes and properties of the elements to solve the problem of mechanical structure design. It is not only able to deal with various problems of material and stress, but also can be applied to the research of heat conduction and other fields, so the finite element analysis method has high flexibility and flexibility [8].

\subsection{Multimedia Visual Analysis Technology}

Multimedia visualization technology is a kind of new technology which is used to convert the data needed by the used of the image processing technology and computer graphics technology [9]. The visual analysis technology is mainly divided into the analysis of computer visualization and information visualization, the former refers to the visualization of spatial data, and the latter refers to the visualization of non spatial data. Visualization technology is mainly to display mufti-dimensional data in three-dimensional space, which provides support for data classification. Currently commonly used data visualization techniques include the matrix method, the technique of visualization, the level of technology, the geometry technique and so on [10].

\section{Methodology}

\subsection{Optimization of Mechanical Structure Size}


In order to optimize the design of the structure of the pipe box mechanical components, this paper makes the structure design of the mechanical components to meet the original design requirements on the basis of the computer programming and mathematical programming theory, and the best technical indicators at the same time. In the design of the variable, the function of the objective function and the constraint condition is needed. Objective function and performance constraint is a complex and invisible function relation based on the basic theory of finite element. In the construction of the parametric finite element analysis model of mechanical components, the model needs to ensure the accuracy and the density of the division, which has a very important role in the optimization of the whole structure design. In addition, in order to obtain the relevant components of the state variables and objective function value, the existing finite element software is used to carry out the finite element analysis to the mechanical components. This paper makes use of the limit element analysis method and the traditional optimization technique to optimize the structure of mechanical parts. The optimization process based on finite element analysis is shown in Fig. 1.

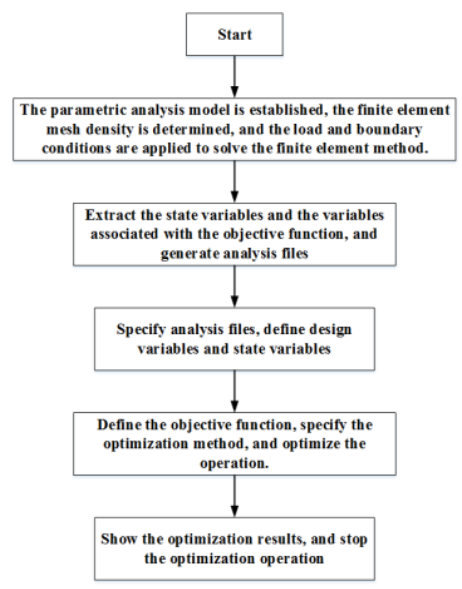

Fig. 1. Optimization process based on finite element analysis

The mathematical model of the size optimization of the pipe box mechanical components can be expressed as:

$$
\left\{\begin{array}{c}
\min _{x \in R^{n}} f(X) \\
\text { s.t. } \\
g_{u}(X) \leq 0,(u=1,2, \ldots, m) \\
h_{q}(X)=0,(q=1,2, \ldots, l<n)
\end{array}\right.
$$

In the formula, $X=\left[x_{1}, x_{2}, \ldots, x_{n}\right]$ represents a design variable, $g_{u}(X)$ and $h_{q}(X)$ represent constraints, and $f(X)$ represents the objective function.

\subsection{Optimization of mechanical structure shape}


Structure characteristics of mechanical components have a decisive role in service life and performance of parts, the optimization of the structural shape is not only conducive to the improvement of its performance, but also increases the utilization rate and the service life of the components. In the design of mechanical structure shape, this paper uses the geometric parameters as the design variables, and uses the area coefficient as the optimization objective function. In addition, the constraint conditions are mainly designed for the performance of the parts. Because the objective function and the constraint condition are the invisible function of the design variables, this paper uses the finite element analysis theory to solve the problem. In addition, in order to solve the problems of mesh deformation even distortion caused by the change of the shape of the components, the grid distortion checking in the whole process of the whole mechanical structure shape optimization is made in this paper. The shape optimization design model can be expressed by the following formula:

$$
\left\{\begin{array}{c}
\text { MinimizeF }(t), i n \Omega \\
\text { s.t.g } g_{i}(t) \leq 0, i=1,2, \ldots, q_{c} \\
t_{j}^{L} \leq t_{j} \leq t_{j}^{U}, j=1,2, \ldots, m \\
\Gamma(t)=\partial \Omega \in R
\end{array}\right.
$$

In the formula, $\mathrm{F}(\mathrm{t})$ is the structural stress, $\mathrm{t}$ is a vector of design variables, $\Omega$ is design domain, $\Gamma(t)$ is the design domain boundary representation, $g_{i}(t)$ is the i-Th constraint condition; $t_{j}^{L}, t_{j}^{U}$ represents the $\mathrm{j}$-Th design variables the upper boundary and the lower boundary, $\mathrm{R}$ is the set of a function.

\section{Result Analysis and Discussion}

In order to verify the validity of this research, the simulation experiment is carried out in this paper. In the process of the experiment, the first order method is used to carry out 10 iterations, and the optimization results are shown in Fig. 2.

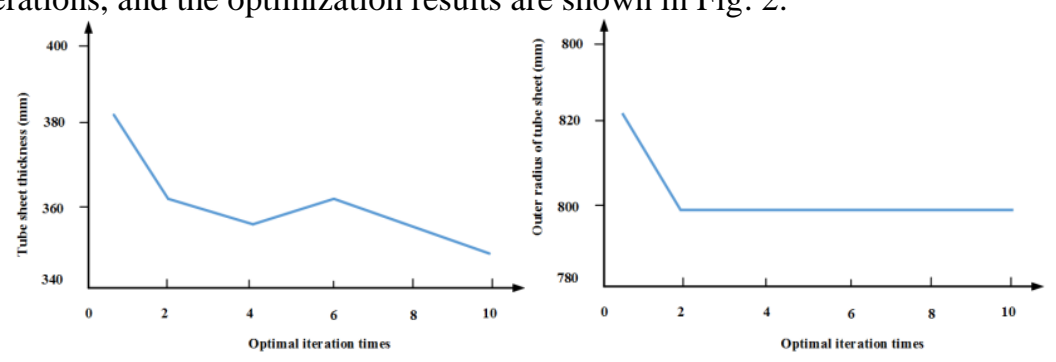

Fig. 2. Optimization results

As can be seen from the above table, when the thickness of the tube plate is about $350 \mathrm{~mm}$ and the outer radius is about $800 \mathrm{~mm}$, the weight of the tube sheet is the smallest. According to the data, In the design of the tube plate structure, the thickness of the tube plate is arranged on the $350 \mathrm{~mm}$, and the radius is $800 \mathrm{~mm}$, which not only can reduce the weight of the mechanical 
structure, but also save the raw materials. In general, the method of the structural design of pipe box mechanical components based on the finite element analysis and multimedia visual analysis technology can improve the overall size and shape of parts, reduce material application, so as to reduce the cost, and realize the computer aided design of mechanical parts.

\section{Conclusion}

Structural design and optimization of mechanical components have a vital role in the economic efficiency and sustainable development of the enterprises. Based on the computer programming and mathematical programming theory, the existing finite element software is used to carry out the finite element analysis to the mechanical components, then the state variables and objective function value of the relevant parts are obtained. In order to make the components achieve performance requirements and optimize the its size as maximize as possible, this paper makes use of the limit element analysis method and the traditional optimization technique to optimize the structure of mechanical components. In addition, in order to solve the problems of mesh deformation even distortion caused by the change of the shape of the components, the grid distortion checking in the whole process of the whole mechanical structure shape optimization is made in this paper. Experimental results show that the scheme proposed in this paper can improve the overall size and shape of the components, reduce material application and the cost, and realize the computer aided design of mechanical parts and the automation of parts design.

\section{References}

[1] Yang Xiaojing, Chen Zichen, Liu Jianxiong, et al. Optimization design of key parts of XK640 CNC milling machine based on ANSYS static stiffness analysis. machine tool and hydraulic, Vol 35, No. 9, pp. 42-45. (2007)

[2] Liu Zhifeng, Yang Ming, Zhang Lei. Research on the design of the detachable connection structure based on. TRIZ China Mechanical Engineering, No. 7, pp. 852-859. (2010)

[3] Wang Xingang, Wang Baoyan, Zhang Kuixiao, et al. Sensitivity design of graded reliability based on measured information. Journal of agricultural engineering, Vol. 28, No. 10, pp. 65-69. (2012)

[4] Xu Huajian, Huang Qiong. Three dimensional design of parameterized 3D design of spur gear based on. Pro/E coal mine machinery, Vol. 35, No. 11, pp. 35-37. (2014)

[5] Zhang Hongjun, Shang Shang. Optimization of packaging machinery components based on Pro/E behavior modeling technology. packaging engineering, Vol. 36, No. 9, pp. 95-98. (2015)

[6] Yi B, Liu Z, Duan G, et al. Finite Element Method and Sharp Features Enhanced Laplacian for Interactive Shape Design of Mechanical Parts. Journal of Computing and Information Science in Engineering, Vol. 14, No. 2, pp. 021, 007. (2014)

[7] Liang Y, Chen W, Sun Y, et al. A mechanical structure-based design method and its implementation on a fly-cutting machine tool design. The International Journal of Advanced Manufacturing Technology, Vol. 70, No. 9-12, pp. 1915-1921. (2014)

[8] Qiu L, Sun L, Liu X, et al. Material selection combined with optimal structural design for mechanical parts. Journal of Zhejiang University SCIENCE A, Vol. 14, No. 6, pp. 383-392. (2013)

[9] Wen-zhong Z. SolidWorks based on the mechanical parts of structure design and application. Manufacturing Automation, Vol. 4, pp. 043. (2012) 
[10] Liu J, Chen B C, Ji H H, et al. Mechanical structure design and application for PCB detection system. Applied Mechanics and Materials. Trans Tech Publications, Vol. 670, pp. 1416-1420. (2014) 